\title{
Research Progress of Cardiac Myosin Binding Protein C in Dilated Cardiomyopathy and Other Cardiac Conditions
}

\author{
Yali Sun', Muhammad Nabeel Dookhun'1,2, Huayiyang Zou', Xiaoqian Cao'1, Yi Zhang', \\ Xin-Zheng Lu1,2* \\ ${ }^{1}$ Department of Cardiology, The First Affiliated Hospital of Nanjing Medical University, Nanjing, China \\ ${ }^{2}$ Department of Cardiology, Sir Run Run Hospital of Nanjing Medical University, Nanjing, China \\ Email: *xzlu@njmu.edu.cn
}

How to cite this paper: Sun, Y.L., Dookhun, M.N., Zou, H.Y.Y., Cao, X.Q., Zhang, Y. and Lu, X.-Z. (2018) Research Progress of Cardiac Myosin Binding Protein C in Dilated Cardiomyopathy and Other Cardiac Conditions. World Journal of Cardiovascular Diseases, 8, 452-461.

https://doi.org/10.4236/wjcd.2018.89044

Received: June 23, 2018

Accepted: September 11, 2018

Published: September 14, 2018

Copyright (c) 2018 by authors and Scientific Research Publishing Inc. This work is licensed under the Creative Commons Attribution International License (CC BY 4.0).

http://creativecommons.org/licenses/by/4.0/

c) (i) Open Access

\begin{abstract}
Since its discovery, myosin-binding protein $\mathrm{C}(\mathrm{cMyBP}-\mathrm{C})$ has become a protein of interest clinically. With emergence of new methodologies and technologies, the structure and functions of $\mathrm{cMyBP}-\mathrm{C}$ from different aspects can be studied, enabling us to better understand its involvement in certain cardiac conditions. Studying its kinetics of release and clearance from the circulation and by comparing to other conventional biomarkers, it has been reported that cMyBP-C is eligible to be a novel biomarker for several cardiac conditions. Moreover, studying the genetics and their involvement in pathogenic mechanisms has opened the ideas for potential therapeutic strategies. More and more researches are constantly being done to better understand the role of cMyBP-C in dilated cardiomyopathy (DCM). The importance of cMyBP-C to the heart is still actively being investigated. Its presence is however crucial for sarcomere organization and proper regulation of cardiac contraction during systole and complete relaxation during diastole. Genetic mutation in cMyBP-C has been linked to cardiac conditions including hypertrophic and dilated cardiomyopathies. Around 350 types of mutations have already been documented leading to various cardiac conditions and abnormalities. Analyzing human heart samples has enabled us to better understand the importance of $\mathrm{cMyBP}-\mathrm{C}$ and how its mutations lead to inherited cardiomyopathies. It is therefore necessary to have an update about the research progress of cMyBP-C in relation to DCM and other cardiac conditions.
\end{abstract}

\section{Keywords}

Dilated Cardiomyopathy, Cardiac Myosin Binding Protein C, Contraction, Diastole, Systole 


\section{Introduction}

Dilated cardiomyopathy (DCM) is the most common type of cardiomyopathies seen clinically. Its pathogenesis is not yet fully understood. Its occurrence and development may be attributed to secondary causes such as viral infection, hypertension, autoimmune cause, alcohol intake and drugs. It has been reported that the 5-year survival rate of DCM is less than 50\% after the emergence of DCM symptoms [1] [2]; in China, the prevalence rate is 19 per 100,000 [3]. Pathologically, DCM changes are characterized by a triad of myocyte degradation, myocyte hypertrophy and myocardial fibrosis [4]. DCM can also be due to idiopathic causes, which is principally due to gene mutations in specific cardiac proteins [5] [6]. The main clinical manifestations of DCM patients are chest tightness, palpitation, dyspnea and decreased activity tolerance, serious symptoms of heart failure in more severe cases, and even appearance of serious heart arrhythmias. Transplantation is the only effective treatment. Current treatment methods are only to improve the symptoms of heart failure and to prevent complications. DCM mainly manifests as a declination of systolic and diastolic functions. There are a wide variety of structural cardiac proteins involved in myocardial contraction and relaxation. One protein of great interest is cMyBP-C. The latter is an important regulotory component of the sarcomere which ensures proper regulation of heart systolic and diastolic function. In this review, we emphasize on an update about the research progress of $\mathrm{cMyBP}-\mathrm{C}$ and its relation to DCM and other cardiac conditions.

\section{Biological Characteristics of cMyBP-C}

\subsection{Classification of $\mathrm{cMyBP}$}

The normal myocardial fibers are composed of regular arrangement of myofibrils that consist of thick myosin and thin actin filaments. The normal systolic and diastolic function of the myocardium depends on the proper interactions of the myofilaments. In addition to myofilaments, myocardium also needs some accessory proteins to help in stabilization of the sarcomere and in cross-bridges formation. The thick filaments are composed of myosin while thin filaments are mainly composed of actin, tropomyosin and troponin. Genetic mutation encoding sarcomere proteins can lead to related myocardial diseases, such as hypertrophic cardiomyopathy (HCM) and dilated cardiomyopathy (DCM).

Myosin binding protein $\mathrm{C}$, being cardiac specific, has lately become of much interest clinically. MyBP-C is encoded by the MyBPC3 gene and is different from the other isoforms encoded by MyBPC1 and MyBPC2 genes (See Table 1).

Table 1. Different Isoforms of MyBPC genes.

\begin{tabular}{cc}
\hline Gene & Expressed in \\
\hline MyBPC1 & Slow skeletal muscle \\
MyBPC2 & Fast skeletal muscle \\
MyBPC3 & Cardiac muscle \\
\hline
\end{tabular}


The myocardium type (C3), also known as human cardiac muscle myosin binding protein $\mathrm{C}$, is specifically expressed in cardiomyocytes, whereas $\mathrm{C} 1$ and $\mathrm{C} 2$ types are only present in skeletal muscle and are not detectable in the myocardium [7].

In this review we will emphasize about the research progress of cardiac myosin-binding protein $\mathrm{C}$ which is an important regulatory protein of the sarcomere.

\subsection{Structure Composition of cMyBP-C}

cMyBP-C was first discovered in the 1970s [8], and it has quickly become a protein of interest clinically. Studies have been done to further understand the structure and functions of $\mathrm{cMyBP}-\mathrm{C}$. This has enabled us to better elucidate its involvement in pathogenesis of certain cardiac conditions. Its gene encodes 1274 amino acids, so cMyBP-C is a large protein with a molecular weight of 140 - 150 $\mathrm{KD}\left(\approx 1.4 \times 10^{4}-1.5 \times 10^{4}\right)$. The protein has 11 functional regions (C0 - C10), of which 8 IgC2 motifs and 3 fibronectin 3 (FNH3) motifs. The "M-domain", also known as the motif, is long polypeptide linkers found between $\mathrm{C} 1$ and $\mathrm{C} 2$ domains. It contains multiple phosphorylation sites, mainly four highly conserved serines (S273, S282, S302, and S307) [9] [10]. These serines are phosphorylated via $\beta$-Adrenergic-stimulation and are believed to regulate cross-bridges formation, thus important for cardiac contractility [11] [12]. cMyBP-C is significantly different from other isoforms of MyBP-C; 1) cMyBP-C contains an Ig assembly of 101 amino acids at the $\mathrm{N}$-terminus (C0). This structure is unique and highly specific to the myocardium; 2) There are four phosphorylation sites in the 105 amino acid-linkage region between $\mathrm{C} 1-\mathrm{C} 2$; 3 ) There is a 28 -amino acids residue domain in the C5 Ig domain [13], which is rich in proline.

\section{Biological Function of cMyBP-C}

\subsection{Phosphorylation of cMyBP-C}

The normal structure of $\mathrm{cMyBP}-\mathrm{C}$ protein is extremely important for their corresponding functions, especially for the correct composition of the myocardium, the contraction and relaxation of the heart. It is known that cMyBP-C has 17 phosphorylation sites, 4 of which are more active (M-domain) [14], mainly distributed between C1-C2. Protein kinase A (PKA) promotes the phosphorylation of $\mathrm{cMyBP}-\mathrm{C}$ through stimulation of Beta-adrenergic agonists [15], resulting in positive inotropic cardiac effects. Large level of intracellular calcium ions released from the sarcoplasmic reticulum binds to $\mathrm{TnC}$ of Troponin complex and $\mathrm{TnI}$ is inhibited. The $\mathrm{C} 1$ component of the $\mathrm{cMyBP}-\mathrm{C}$ binds to induce a conformational change and causes a shift in the tropomyosin complex [16]. This activates the thin filament into an open structural state and exposes the binding site of actin. Hydrolysis of ATP on the myosin induces cross-bridges formation between myosin head and active site on actin [17]. The strength of contraction is said to be dependent on the number of cross-bridges formed [12]. 


\section{2. cMyBP-C Regulates Systolic Contraction and Diastolic Relaxation}

The systolic and diastolic function of the heart muscle depends on the correct interaction of the sarcomeric proteins. The contraction mechanism of the myocardium is from the release of energy of the thick filament proteins through the cross-bridges with ATPase activity and AIPse, which in turn causes the cross-bridges to interact with the thin filaments to produce movement. Hofmann et al. at first reported the important function of cMyBP-C in the 1990s [18]. Phosphorylation of cMyBP-C can affect the dynamic changes of the cross-bridges formation, which in turn regulates the myosin-actin interaction. The force developed within the myocardium relies on the rate at which cross-bridges are recruited [19]. The phosphorylation of cMyBP-C is regulated by intracellular calcium ion concentrations and beta-adrenergic agonists, which in turn regulates the action of myosin S2 [20]. Phosphorylated cMyBP-C has a reduced affinity to myosin and does not bind to myosin S2. This in turns enhances the interaction between myosin and actin [21] and promotes the contraction of the myocardium. It is the number of cross-bridges that ultimately determines the peak pressure achieved [22].

The removal of calcium ions from the cytoplasm initiates the process of relaxation. Other processes including the rate of thin filament deactivation and rate of cross-bridges detachment are also involved [23]. It is generally believed that $\mathrm{cMyBP}-\mathrm{C}$ exerts a greater influence on the diastolic function than on the contractile function of the myocardium. Animal studies [19] have demonstrated that $\mathrm{cMyBP}-\mathrm{C}$ plays an extremely important role in myocardial diastolic function. Studies showed that $\mathrm{cMyBP}-\mathrm{C}$ phosphorylation is an important mediator of diastolic function and increases the rate of cross-bridges detachment, thus ensuring proper relaxation [24]. Impaired phosphorylation of cMyBP-C can lead to diastolic dysfunction as seen in hypertrophic cardiomyopathy (HCM) [25]. Impaired $\mathrm{cMyBP}-\mathrm{C}$ phosphorylation leads to slow deactivation of thin filament and slow the rate of detachment of cross-bridges from the thin filament. Consequently, ventricular relaxation will be affected and prolonged. Therefore, cMyBP-C phosphorylation accelerates rate of cross-bridges detachment, thus ensuring relaxation for proper diastolic function.

\section{4. cMyBP-C and Cardiac Conditions}

\section{1. cMyBP-C and Hypertrophic Cardiomyopathy}

Since $\mathrm{CMyBP}-\mathrm{C}$ has a great influence on both the diastolic and systolic function of the myocardium, any abnormality of $\mathrm{CMyBP}-\mathrm{C}$ or mutation of its coding gene will definitely have a certain influence on the function of the myocardium. The most frequently mutated gene in HCM is perhaps the cMyBP-C gene [26]. cMyBP-C has gain so much attention clinically since the discovery that cMyBP-C gene mutation can lead to HCM [27]. Watkins et al. [28] and Bonne et al. [29] respectively reported that $\mathrm{cMyBP}-\mathrm{C}$ gene deletion, insertion, and mis- 
sense mutations might damage the structure and functional integrity, leading to hypertrophic cardiomyopathy. More and more mutation sites of cMyBP-C pathogenic genes have been found, and about 350 have been reported so far [30]. Animal model have proven that $\mathrm{cMyBP}-\mathrm{C}$ mutations lead to hypertrophic cardiomyopathy. Yang et al. [31] constructed truncated protein models of which one expresses a truncated protein and the other one a myosin-binding site that lacks myosin and myosin binding sites. Both animal models eventually showed similar hypertrophic myocardium. Different domains of the cMyBP-C can undergo different type of mutation and thus leading to different structural or dysfunction of the cMyBP-C protein. For example, a Japanese study showed that a frame-shift deletion in MyBP-C lead to left ventricular remodeling of HCM [32]. HCM with left ventricular dysfunction and dilation in elderly is due to a missense mutation on MyBP-C as shown by another study [33].

\section{2. cMyBP-C and Dilated Cardiomyopathy}

Genetic mutations of cMyBP-C are not only related to hypertrophic cardiomyopathy. Recent studies have found that cMyBP-C genetic defects can also lead to dilated cardiomyopathy (DCM) [33], such as the Asn948Thr missense mutation [34]. A multinational study of 639 patients with dilated cardiomyopathy showed that the $\mathrm{cMyBP}-\mathrm{C}$ gene mutation is the second highest mutated gene [35]. cMyBP-C may also be involved in the pathogenesis of DCM though eliciting an autoimmune response [36]. Post myocardial infarction causes proteolysis of $\mathrm{C} 0 \mathrm{C} 1$ fragment of $\mathrm{CMyBP}-\mathrm{C}$ and result in production of auto-antibodies which is believed to induce the onset of autoimmune myocarditis and hence ultimately progress to DCM and heart failure [37]. Studies have shown the presence of auto-antibodies to onset of autoimmune myocarditis and DCM [38].

\section{5. cMyBP-C as Cardiac Biomarkers}

\section{1. cMyBP-C as Biomarker for Myocardial Infarction}

Cardiac Troponin (cTn) has long been the preferred serum biomarker for the diagnosis of acute myocardial infarction (AMI) [39], but it is also sensitive to other diseases such as myocarditis, severe pulmonary infection, and pulmonary embolism [40]. Myocardial injury requires quick attention and better biomarkers which are more specific than cTn are still needed. cMyBP-is among the most voluminous cardiac protein quantified, twice as much as cTnI and cTnT [41].

cTn gradually increases after 4 to 6 hours post-MI, and is unable to be detected upon early onset of myocardial injury. Studies have been done to understand the kinetics of release and clearance of cMyBP-C as compared to cTnT. It turns out that cMyBP-C, as a biomarker, rises and clears more rapidly as compared to cTnT [42] [43]. Govindan et al. [44] pointed out that cMyBP-C can be a new marker of myocardial necrosis. Its serum concentration is several times higher than cTn and it is easy to be detected. Kuster et al. [45] experiments 
showed that serum $\mathrm{CMyBP}-\mathrm{C}$ concentration began to increase significantly after 30 minutes post-MI after ligation of porcine coronary arteries. Another clinical study [46] confirmed the observation that serum cMyBP-C in patients with acute myocardial infarction was significantly higher than the traditional markers of myocardial necrosis, and began to increase within 4 hours of onset; and was significantly reduced in serum after 12 hours of emergency stenting surgery. This indicates that serum cMyBP-C can be used as a biochemical marker for the early diagnosis of AMI.

\section{2. cMyBP-C as Biomarker for DCM and Heart Failure}

Dilated Cardiomyopathy is a common cause of heart failure, affecting the heart musculature and vasculature and involves one or several underlying pathophysiological mechanisms [47]. Myocyte injury can lead to DCM and causes release of related biomarkers including that of cMyBP-C. At cutoff value of $45 \mathrm{ng} / \mathrm{ml}$, serum $\mathrm{CMyBP}-\mathrm{C}$ turns out to have $100 \%$ sensitivity and $96 \%$ specificity as diagnostic biomarker for heart failure [48]. The exact release mechanism of cMyBP-C in heart failure is still unknown. Moreover, cMyBP-C has a $90 \%$ sensitivity and $93 \%$ specificity as a biomarker of prognosis in heart failure patient at cutoff value of $152 \mathrm{ng} / \mathrm{ml}$ [48]. The same study showed that failure to decrease the level of serum $\mathrm{cMyBP}-\mathrm{C}$ leads to poor prognosis. Therefore, MyBP-C has both the characteristics of being diagnostic and prognostic biomarker.

\section{Conclusion}

With the emergence of new research methodologies and technologies, great progress has been made towards understanding the structure and function of sarcomeric proteins. The structure and physiological functions of $\mathrm{cMyBP}-\mathrm{C}$ are now relatively clearer. $\mathrm{cMyBP}-\mathrm{C}$ has become a protein of interest due to its potential use clinically. Understanding the structure and functions of $\mathrm{cMyBP}-\mathrm{C}$ enables us to better relate its involvement in pathogenesis of certain cardiac conditions including that of cardiomyopathies and heart failure. Studying its release, kinetics and clearance from the circulation has demonstrated than it can be used as a novel biomarker for AMI. Its presence is however crucial for sarcomere organization and proper regulation of cardiac contraction during systole and complete relaxation during diastole. Genetic mutation in $\mathrm{cMyBP}-\mathrm{C}$ has been linked to several cardiac conditions. Around 350 types of mutations have already been documented. Moreover, prompt understanding of $\mathrm{cMyBP}-\mathrm{C}$ in term of structure, genetics and functions, is important and can be insightful in terms of therapeutic strategies towards HCM, DCM, heart failure, AMI and other related cardiac conditions.

\section{Conflicts of Interest}

The authors declare no conflicts of interest regarding the publication of this paper. 


\section{References}

[1] Grogan, M., et al. (1995) Long-Term Outcome of Patients with Biopsy-Proved Myocarditis: Comparison with Idiopathic Dilated Cardiomyopathy. Journal of the American College of Cardiology, 26, 80-84. https://doi.org/10.1016/0735-1097(95)00148-S

[2] Komajda, M. and Jais, J.P. (1990) Factors Predicting Mortality in Idiopathic Dilated Cardiomyopathy. European Heart Journal, 11, 824-831. https://doi.org/10.1093/oxfordjournals.eurheartj.a059803

[3] Wang, Z.M., Zou, T.B., Song, L., et al. (2004) Echocardiographic Examination of the Prevalence of Dilated Cardiomyopathy in 8080 Adults. Chinese Journal of Cardiovascular Diseases, 32, 1090-1094.

[4] Davies, M.J. and Mckenna, W.J. (1994) Dilated Cardiomyopathy: An Introduction to Pathology and Pathogenesis. British Heart Journal, 72, S24.

[5] McNally, E.M., Golbus, J.R. and Puckelwartz, M.J. (2013) Genetic Mutations and Mechanisms in Dilated Cardiomyopathy. Journal of Clinical Investigation, 123, 19-26. https://doi.org/10.1172/JCI62862

[6] Garciapavia, P., et al. (2013) Genetics in Dilated Cardiomyopathy. Biomarkers in Medicine, 7, 517-533. https://doi.org/10.2217/bmm.13.77

[7] Oakley, C.E., Chamoun, J., Brown, L.J., et al. (2001) Myosin Binding Protein C: Enigmatic Regulator of Cardiac Contraction. The International Journal of Biochemistry \& Cell Biology, 39, 2161-2166. https://doi.org/10.1016/j.biocel.2006.12.008

[8] Craig, R. and Offer, G. (1976) The Location of C-Protein in Rabbit Skeletal Muscle. Proceedings of the Royal Society B, 192, 451-461. https://doi.org/10.1098/rspb.1976.0023

[9] Nabeel, D., Zhang, J.-N. and Lu, X.-Z. (2017) MyBP-C in Cardiac Conditions and Its Potential Use as Novel Biomarker: A Review. European Academic Research, 5, 1352-1368.

[10] Kuster, D.W.D., et al. (2013) GSK3 $\beta$ Phosphorylates Newly Identified Site in the Proline-Alanine-Rich Region of Cardiac Myosin-Binding Protein C and Alters Cross-Bridge Cycling Kinetics in Human: Short Communication. Circulation Research, 112, 633-639.

[11] Palmer, B.M., et al. (2011) Roles for Cardiac MyBP-C in Maintaining Myofilament Lattice Rigidity and Prolonging Myosin Cross-Bridge Lifetime. Biophysical Journal, 101, 1661-1669. https://doi.org/10.1016/j.bpj.2011.08.047

[12] Gresham, K.S. and Julian, E.S. (2016) The Contributions of Cardiac Myosin Binding Protein $\mathrm{C}$ and Troponin I Phosphorylation to $\beta$-Adrenergic Enhancement of in $\mathrm{Vi}$ vo Cardiac Function. The Journal of Physiology, 594, 669-686.

https://doi.org/10.1113/JP270959

[13] Gautel, M., Zuffardi, O., Freiderg, A., et al. (1995) Phosphorylation Switches Specific for the Cardiac Isoforms of Myosin Binding Protein C: A Modulator of Cardiac Contraction. The EMBO Journal, 14, 1952-1960. https://doi.org/10.1002/j.1460-2075.1995.tb07187.x

[14] Jia, W., Shaffer, J.F., Harris, S.P., et al. (2010) Identification of Novel Protein Kinase A Phosphorylation Sites in the M-Domain of Human and Murine Cardiac Myosin Binding Protein-C Using Mass Spectrometry Analysis. Journal of Proteome Research, 9, 1843-1853. https://doi.org/10.1021/pr901006h

[15] Venema, R.C. and Kuo, J.F. (1993) Protein Kinase C-Mediated Phosphorylation of Troponin I and C-Protein in Isolated Myocardial Cells Is Associated with Inhibition 
of Fibrillar Actomyosin MgATPase. Biophysical Journal, 268, 2705-2711.

[16] Walcott, S., Steffen, D. and Samantha, P.H. (2015) Effects of Cardiac Myosin Binding Protein-C on Actin Motility Are Explained with a Drag-Activation-Competition Model. Biophysical Journal, 108, 10-13. https://doi.org/10.1016/j.bpj.2014.11.1852

[17] Xie, P. (2010) A Model for Processive Movement of Single-Headed Myosin-IX. Biophysical Chemistry, 151, 71-80. https://doi.org/10.1016/j.bpc.2010.05.007

[18] Hofmann, P.A., Greaser, M.L. and Moss, R.L. (1991) C-Protein Limits Shortening Velocity of Rabbit Skeletal Muscle Fibres at Low Levels of $\mathrm{Ca}^{2+}$ Activation. The Journal of Physiology, 439, 701-715. https://doi.org/10.1113/jphysiol.1991.sp018689

[19] Moss, R.L. and Daniel, P.F. (2010) Regulation of Contraction in Mammalian Striated Muscles-The Plot Thickens. The Journal of General Physiology, 136, 21-27. https://doi.org/10.1085/jgp.201010471

[20] Flashman, E., Redwood, C., Moolman-Smook, J., et al. (2004) Cardiac Myosin Binding Protein C: Its Role in Physiology and Disease. Circulation Research, 94, 1279-1289. https://doi.org/10.1161/01.RES.0000127175.21818.C2

[21] Weisberg, A. and Winegrad, S. (1996) Alteration of Myosin Cross Bridges by Phosphorylation of Myosin-Binding Protein C in Cardiac Muscle. Proceedings of the National Academy of Sciences of the United States, 93, 8999-9003. https://doi.org/10.1073/pnas.93.17.8999

[22] Hinken, A.C. and Solaro, R.J. (2007) A Dominant Role of Cardiac Molecular Motors in the Intrinsic Regulation of Ventricular Ejection and Relaxation. Physiology, 22, 73-80. https://doi.org/10.1152/physiol.00043.2006

[23] Bilchick, K.C., et al. (2007) Heart Failure-Associated Alterations in Troponin I Phosphorylation Impair Ventricular Relaxation-Afterload and Force-Frequency Responses and Systolic Function. American Journal of Physiology-Heart and Circulatory Physiology, 292, 318-325.

[24] Rosas, P.C., et al. (2015) Phosphorylation of Cardiac Myosin-Binding Protein-C Is a Critical Mediator of Diastolic Function. Circulation-Heart Failure, 8, 582-594. https://doi.org/10.1161/CIRCHEARTFAILURE.114.001550

[25] Frey, N., Mark, L. and Hugo, A.K. (2012) Mechanisms of Disease: Hypertrophic Cardiomyopathy. Nature Reviews Cardiology, 9, 91-100. https://doi.org/10.1038/nrcardio.2011.159

[26] Schlossarek, S., Giulia, M. and Lucie, C. (2011) Cardiac Myosin-Binding Protein C in Hypertrophic Cardiomyopathy: Mechanisms and Therapeutic Opportunities. Journal of Molecular and Cellular Cardiology, 50, 613-620. https://doi.org/10.1016/j.yjmcc.2011.01.014

[27] Yu, B., French, J.A., Carrier, L., Jeremy, R.W., et al. (1998) Molecular Pathology of Familial Hypertrophic Cardiomyopathy Caused by Mutations in Myosin Binding Protein C. Journal of Medical Genetics, 35, 205-210. https://doi.org/10.1136/jmg.35.3.205

[28] Watkins, H., Conner, D., Thierfelder, L., et al. (1995) Mutations in the Cardiac Myosin Binding Protein-C Gene on Chromosome 11 Cause Familial Hypertrophic Cardiomyopathy. Nature Genetics, 11, 434-437. https://doi.org/10.1038/ng1295-434

[29] Bonne, G., Carrier, L., Bercovici, J., et al. (1995) Cardiac Myosin Binding Protein-C Gene Splice Acceptor Site Mutation Is Associated with Familial Hypertrophic Cardiomyopathy. Nature Genetics, 11, 438-440. https://doi.org/10.1038/ng1295-438

[30] Carrier, L., et al. (2015) Cardiac Myosin-Binding Protein C (MYBPC3) in Cardiac Pathophysiology. Gene, 573, 188-197. https://doi.org/10.1016/j.gene.2015.09.008 
[31] Yang, Q., Sanbe, A., Osinska, H., et al. (1999) In Vivo Modeling of Myosin Binding Protein C Familial Hypertrophic Cardiomyopathy. Circulation Research, 85, 841-847. https://doi.org/10.1161/01.RES.85.9.841

[32] Kubo, T., et al. (2005) Lifelong Left Ventricular Remodeling of Hypertrophic Cardiomyopathy Caused by a Founder Frameshift Deletion Mutation in the Cardiac Myosin-Binding Protein C Gene Among Japanese. Journal of the American College of Cardiology, 46, 1737-1743. https://doi.org/10.1016/j.jacc.2005.05.087

[33] Konno, T., Shimizu, M., Lno, H., et al. (2003) A Novel Missense Mutation in the Myosin Binding Protein C Gene Is Responsible for Hypertrophic Cardiomyopathy with Left Ventricular Dysfunction and Dilation in Elderly Patients. Journal of the American College of Cardiology, 41, 781-786. https://doi.org/10.1016/S0735-1097(02)02957-1

[34] Daehmlow, S., Erdmann, J., Knueppel, T., et al. (2002) Novel Mutations in Sarcomeric Protein Genes in Dilated Cardiomyopathy. Biochemical and Biophysical Research Communications, 298, 116-120. https://doi.org/10.1016/S0006-291X(02)02374-4

[35] Haas, J., Frese, K.S., Peil, B., et al. (2015) Atlas of the Clinical Genetics of Human Dilated Cardiomyopathy. European Heart Journal, 36, 1123-1135.

https://doi.org/10.1093/eurheartj/ehu301

[36] Doesch, A.O., et al. (2010) Impact of Troponin I-Autoantibodies in Chronic Dilated and Ischemic Cardiomyopathy. Basic Research in Cardiology, 106, 25-35. https://doi.org/10.1007/s00395-010-0126-Z

[37] Matsumoto, Y., Ilkwon, P. and Kuniko, K. (2007) B-Cell Epitope Spreading Is a Critical Step for the Switch from C-Protein-Induced Myocarditis to Dilated Cardiomyopathy. American Journal of Pathology, 170, 43-51. https://doi.org/10.2353/ajpath.2007.060544

[38] Kaya, Z., Christoph, L. and Hugo, A.K. (2012) Autoantibodies in Heart Failure and Cardiac Dysfunction. Circulation Research, 110, 145-158. https://doi.org/10.1161/CIRCRESAHA.111.243360

[39] Morrow, D.A., Cannon, C.P., Jesse, R.L., et al. (2007) National Academy of Clinical Biochemistry Laboratory Medicine Practice Guidelines: Clinical Characteristics and Utilization of Biochemical Markers in Acute Coronary Syndromes. Clinical Chemistry, 53, 552-574. https://doi.org/10.1373/clinchem.2006.084194

[40] Gerber, B.L., Rochitte, C.E., Melin, J.A., et al. (2000) Microvascular Obstruction and Left Ventricular Remodeling Early after Acute Myocardial Infarction. Circulation, 101, 2734-2741. https://doi.org/10.1161/01.CIR.101.23.2734

[41] Aye, T., et al. (2010) Proteome-Wide Protein Concentrations in the Human Heart. Molecular BioSystems, 6, 1917-1927. https://doi.org/10.1039/c004495d

[42] Kuster, D.W.D., et al. (2014) Release Kinetics of Circulating Cardiac Myosin Binding Protein-C Following Cardiac Injury. American Journal of Physiology-Heart and Circulatory Physiology, 306, 547-556.

[43] Baker, J.O., et al. (2015) Cardiac Myosin-Binding Protein C: A Potential Early Biomarker of Myocardial Injury. Basic Research in Cardiology, 110, 23-23. https://doi.org/10.1007/s00395-015-0478-5

[44] Bolognese, L., Carrabba, N., Parodi, G., et al. (2004) Impact of Microvascular Dysfunction on Left Ventricular Remodeling and Long-Term Clinical Outcome after Primary Coronary Angioplasty for Acute Myocardial Infarction. Circulation, 109, 1121-1126. https://doi.org/10.1161/01.CIR.0000118496.44135.A7

[45] Nijveldt, R., Beek, A.M., Hirsch, A., et al. (2008) Functional Recovery after Acute 
Myocardial Infarction: Comparison between Angiography, Electrocardiography, and Cardiovascular Magnetic Resonance Measures of Microvascular Injury. Journal of the American College of Cardiology, 52, 181-189.

https://doi.org/10.1016/j.jacc.2008.04.006

[46] Gu, Z.C. (2014) Study on the Role of Serum cMyBP-C and VEGF-B in the Diagnosis and Prognosis of Patients with Acute Myocardial Infarction. Soochow University, Suzhou.

[47] Dookhun, M.N., Sun, Y.L., Zou, H.Y.Y., Cao, X.Q. and Lu, X.Z. (2018) Classification of New Biomarkers of Dilated Cardiomyopathy Based on Pathogenesis-An Update. Health, 10, 300-312. https://doi.org/10.4236/health.2018.103024

[48] El Amrousy, H., et al. (2017) Prognostic Value of Plasma Levels of Cardiac Myosin Binding Protein-C as a Novel Biomarker in Heart Failure. Pediatric Cardiology, 38, 418-424. https://doi.org/10.1007/s00246-016-1532-2 